\author{
Proceedings of the Conference on "Nuclear Structure in the Nineties," \\ Oak Ridge, Tennessee, April 23.27, 1990
}

\title{
STUDY OF SPIN-TEMPERATURE EFFECTS USING ENERGY-ORDERED CONTINUUM GAMMA-RAY SPECTROGCOPY TECHNIQUE
}

\author{
C. Baktash, M.L. Halbert, D.C. Hensley, N.R. Johnson, I.Y. Lee, \\ J.W. McConnell, F.K. McGowan \\ Physics Division, Oak Ridge National Laboratory, Oak Ridge, TN 37891-6371
}

\begin{abstract}
We have investigated a new continuum $\gamma$-ray spectroscopy technique which is based on the detection of all emitted $\gamma$ rays in a $4 \pi$ detector system, and ordering them according to their energies on an event-by-event basis. The technique allows determination of gamma strength functions, and rotational damping width as a function of spin and temperature. Thus, it opens up the possibility of studying the onset of motional narrowing, orcier-to-chaos transition, and the mapping of the evolution of nuclear collectivity with spin and temperature. Application of the technique for preferential entry-state population, exit-channel selection, and feeding of the discrete states via selective pathways vill be discussed.
\end{abstract}

\section{INTRODUCTION}

In the past few years, we have witnessed a rapid progress toward understanding the properties of cold rapidly-rotating nuclei. For example, the spectroscopy of the discrete superdeformed, as well as normally-deformed states in the $50-60 \hbar$ spin range is becoming "routine", thanks to both the ingenuity of the experimentalists, and the advent of powerful gamma-ray detection systems. The picture, unfortunately, is not as bright when it comes to the understanding of the nuclear properties at finite temperature. This lack of knowledge, however, is not due to the want of interesting physics to be addressed in this regime. Quite on the contrary, dramatic structural changes are expected to unfold as the excitation energy is increased. Gradual vanishing of the pairing correlations, onset of chaos, prolate-to-oblate shape changes, melting of the shell effects, and eventually the inability of the compound nucleus (CN) to sustain giant resonances at very high temperatures ("death" of CN) are only a few examples of the rich and varied phenomena yet to be explored. Equally important is the question of thermal fluctuations and the extent to which they modify the above phase transitions.

Heavy-ion fusion-evaporation reactions remain the basic tool to form and study hot nuclei. Unfortunately, many important parameters that influence, or even determine the formation and decay of compound nuclei are not yet reliably known. Among these are the evolution with spin and temperature of the level density, gamma-ray strength functions and transmission coefficients. These parameters determine the competition between neutron, charged-particle, and gamma emission and, thus, critically enter into our Monte Carlo simulations of the CN decay, upon which interpretations of the bulk of the experimental data rest. Naturally, a vigorous theoretical and experimental effort must be undertaken to remedy the situation 
before we can fully explore the various phases of nuclear matter at finite temperature that was outlined above.

At ORNL, we have utilized a variety of complementary probes and experimental techniques to study the nuclear response to the DIFFERENTIAL effects of increasing spin and temperature for constant values of intrinsic excitation energy (U) and spin (I). The focus of the present work is the investigation of a new experimental technique iu nrobe variations of gamma-ray strength functions, and rotational damping width $\left(\Gamma_{\text {rot }}\right)$ with spin and temperature. We have also studied the effects of high-energy $\gamma$-ray gating on the entry-state population, exit-channel selection, and feeding of the yrast line via selective pathways. A preliminary result of these studies are presented in the following sections.

\section{EXPERIMENTAL TECHNIQUE}

This new technique revolves around nearly-complete detection of the deexcitation $\gamma$-rays of compound nuclei, and ordering them according to their energies on an event-by-event basis. For an idea' rigid rotor, energies of the emitted $\gamma$-rays increase linearly with spin. Thus, histograming energies of the 1st, $2 \mathrm{nd}, \ldots$, Nth emitted $\gamma$ rays that belong to the same cascade will result in a "picket fence" of equally-spaced sharp peaks that are separated by $\Delta\left(E_{\gamma}\right)=$ $4 h^{2} / J^{(2)}$. Therefore, for a given rotational band, the energy of each $\gamma$ ray directly reflects its relative position in that cascade, and identifies the spin of the state from which it originated. Motivated by this picture, we will adopt an ordering scheme where the $\gamma$-ray with the highest (lowest) energy will be labeled as the first (last) $\gamma$ ray emitted in the event. (It should be emphasized that this labeling procedure is strictly operational and that, because of the statistical transitions, the first emitted $\gamma$ ray does not have the highest energy in every event.)

However, two factors perturb this simple relationship in realistic nuclei. First, because of the mixing of rotational bands in warm nuclei that sets in at excitation energies of $U \simeq 1$ $2 \mathrm{MeV}$ ( see discussion of rotational damping width in Sec. 5), a mixed state at spin I will decay to an ensemble of states with a nearly Gaussian distribution. Therefore, $\gamma$-ray cascades that result from deexcitation of collective states at finite temperature will produce a picket fence of nearly-Gaussian peaks. Conversely, in an ideal experiment performea vith $100 \%$ detection efficiency, the observed widths of these Gaussian distributions would reflect $\Gamma_{\text {rot }}$. A second confounding factor arises from the competition between the statistical cooling transitions and the collective intraband transitions. Because of the irregularity of the energies of the statistical cooling transitions, they are randomly intersperced between the regular collective transitions. Therefore, they produce exponentially-sloping shoulders that are superimposed on the above Gaussian peaks, and result in skewed distribution functions. From the slopes and intensities of the high-and low-energy shoulders we can infer the cooling rate and the $E 1 / E 2$ ratios of gamma-ray strength functions along the deexcitation pathway. Thus, after correcting for detector response functions, the experimentally obseved deviations from the ideal rigidrotor pattern may be used to deduce important information about nuclear properties at finite temperature.

To do so requires use of $4 \pi$-detector systems that detect nearly all emitted $\gamma$ rays. Moreover, 


\section{DISCLAIMER}

This report was prepared as an account of work sponsored by an agency of the United States Government. Neither the United States Government nor any agency thereof, nor any of their employees, makes any warranty, express or implied, or assumes any legal liability or responsibility for the accuracy, completeness, or usefulness of any information, apparatus, product, or process disclosed, or represents that its use would not infringe privately owned rights. Reference herein to any specific commercial product, process, or service by trade name, trademark, manufacturer, or otherwise does not necessarily constitute or imply its endorsement, recommendation, or favoring by the United States Government or any agency thereof. The views and opinions of authors expressed herein do not necessarily state or reflect those of the United States Government or any agency thereof. 
such detectors are needed to obtain total energy ( $\mathrm{L}$ ), and multiplicity ( $\mathrm{M}$ ) information in order to isolate decay pathways that originate from small regions of the (E,I) map. Comparison of energy-ordered $\gamma$-ray spectra that correspond to decay pathways at constant $M$, and $U$ will then reveal the differential effects of temperature and spin, respectively, on the $E 1 / E 2$ competition, $\Gamma_{\text {rot }}$, and dynamical moments of inertia $J^{(2)}$.

We have applied these ideas to the case of well-deformed ${ }^{170} \mathrm{Hf}$ nucleus which was produced in the ${ }^{130} \mathrm{Te}\left({ }^{44} \mathrm{Ca}, 4 \mathrm{n}\right)$ reaction at $195.5 \mathrm{MeV}$ bearn energy. The experimental setup consisted of the Spin Spectrometer ${ }^{1}$ and the ORNL Compton-Suppression Spectrometer System (18 Ge detectors). The high-resolution $\gamma$ data collected with the Ge detectors have been analyzed to establish both an extensive decay scheme, and to obtain estimates of $\Gamma_{\text {rot }}$ over the range of $E_{\gamma}=700-1200$ using $\gamma-\gamma$ energy correlation technique ${ }^{2}$. The latter information will provide an independent check on the validity of the new technique proposed here.

The NaI elements of the Spin Spectrometer were used to obtain both continuum $\gamma$ ray spectra, and information regarding the total coincidence pulse height $(H)$, and fold $(k)$. Discrete $\gamma$ rays emitted from the low-lying states in ${ }^{170} \mathrm{Hf}$ were used as gates in order to uniquely identify cascades associated with this residual nucleus. Figure 1 shows histograms of the energy-ordered (1st, 2nd, 3rd, 4th, 6th, and 10th) $\gamma$ rays from $k=20$ fold events. (Again, it must be emphasized that what we label as the "first" $\gamma$ ray is different than the so called "first-generation" statistical $\gamma$ ray described in, e.g., Ref. 3.) The slopes of the high-energy statistical tails of these spectra quickly become steeper as we go to the later transitions, clearly reflecting the effects of cooling process. For $\mathrm{N}>5$ (where $\mathrm{N}$ is the ordinal number of the $\gamma$ ray), the distributions become nearly Gaussian, with some statistical tails. Assuming that centroids of these spectra correspond to the average $\gamma$-ray energies, we extracted dynamical moments of inertia, $J^{(2)}$, which are in good agreement with the average values obtained from the high-spin discrete band structures in this nucleus.

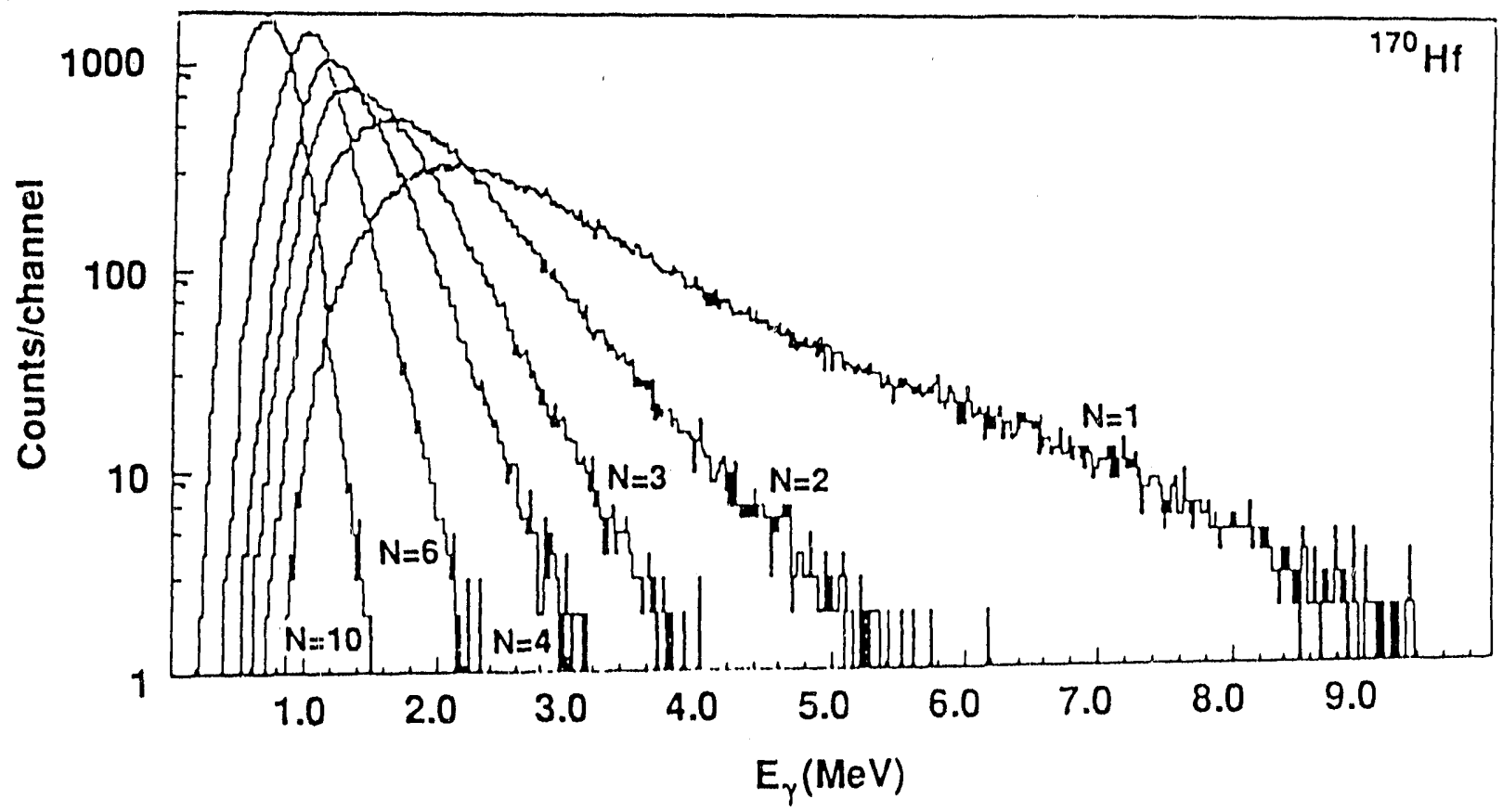

Figure 1: Energy-ordered $\gamma$ rays for $\mathrm{k}=20$ events. 


\section{EFFECTS OF HIGH-ENERGY $\gamma$-RAY GATING ON ENTRY-STATE POP- ULATION AND CHANNEL SELECTION}

In order to gain maximum sensitivity in their measurements, experimental nuclear physicists continously strive to achieve as much control on event selection as possible. This is usually done by construction of more powerful detectors, and refinements of experimental techniques. In nuclear-structure studies of compound nuclei, construction of $4 \pi$ detectors (such as the Spin Spectrometer) was a major step forward in this direction. By providing information on $\mathrm{H}$ (proportional to total excitation energy, E) and k (proportional to spin), the Spin Spectrometer offers a significant filtering capability. For example, even if the entry distribution of the different residual nuclei overlap to some extent, a significant degree of exit-channel selection is achieved by the so called $(\mathrm{H}, \mathrm{k})$ gating technique. Additional selectivity requires use of other independent observables that would remove the $(\mathrm{H}, \mathrm{k})$ degeneracy in the overlapping region. Here, we shall examine the effectiveness of high-energy statistical $\gamma$ rays, which reflect the intrinsic excitation energy $(U)$ of the $\mathrm{CN}$, to provide selectivity for temperature, entry distribution and channel selection. For the purpose of this study, we have summed up the energies of the first two $\gamma$ rays to reduce the effects of less-than-complete detection efficiency. We shall denote this quantity as $E_{\gamma_{1}+\gamma_{2}}$.

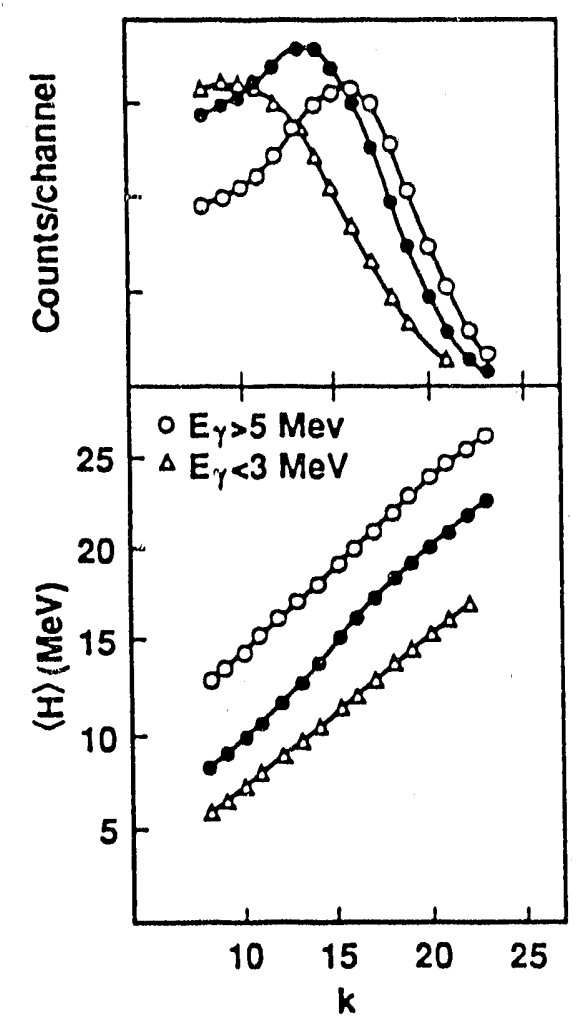

Figure 2: Entry-State Distribution $\langle H\rangle$ as a function of the fold $(\mathrm{k})$ with gates set on the high-energy gamma: $E_{\gamma}<3 \mathrm{MeV}$ (triangles), and $E_{\gamma}>5 \mathrm{MeV}$ (open circles). The filled circles show the total entry-state distribution. The top panel shows the corresponding fold distributions.

\subsection{Temperature and entry-distribution selection}

Studies of feeding mechanisms into high-spin yrast states (both normal and superdeformed), and of temperature effects on nuclear structure require the ability to select the temperature of entry distributions. As shown in Fig. 2, this may be achieved by gating on the high-energy $\gamma$ rays. The entry lines corresponding to a low $(<3 \mathrm{MeV})$, and high $(>5$ 
$\mathrm{MeV}$ ) gates on $E_{\gamma^{1+\gamma^{2}}}$ (triangles and open circles, respectively) are separated by $\simeq 5-6 \mathrm{MeV}$ over a wide range of $k$. The ungated entry line (filled circles) t'rat reflects the total entry distribution falls roughly midway between the two extremes. The effects of these gates on the k-distribution (top panel of Fig. 2) amount to a centroid shift of nearly six transitions. Thus, high- $E_{\gamma}$ gating provides a simple and very effective filter of the temperature of the entry distribution, and may be used to isolate regions of different excitation energy to study nuclear response at finite temperature.

\subsection{Exit-channel selection and high-spin yrast state feeding}

Because of the ability of high- $E_{\gamma}$ gating to lift the $(\mathrm{H}, \mathrm{k})$ degeneracy by nearly one neutron B.E., it provides a simple and effective filter for exit-channel selection either aloue or in conjunction with $(\mathrm{H}, \mathrm{k})$ gating. Figure 3 shows Ge-spectra obtained subject to high (panel $\mathrm{c}$ ), and low (panel b) $E_{\gamma_{1}+\gamma_{2}}$ gates. While $\gamma$ rays in the $4 \mathrm{n}$ channel ${ }^{170} \mathrm{Hf}$, whose production is maximized in this reaction, dominate the spectrum in panel (c), the yield of the $5 \mathrm{n}$ channel leading to ${ }^{160} \mathrm{Hf}$ significantly exceeds that of ${ }^{170} \mathrm{Hf}$ in panel (b). Additional use of k-gating would further increase this selectivity.

Because of the interest in the study of very high-spin discrete states, it is important to devise gating techniques that would enhance feeding of these states. Since collective E2 strength persisto at high excitation energies, and carries some of the $\gamma$-ray flow parallel to the yrast line, cascades that originate from hot entry distributions reach the near-yrast states at lower spins compared to those that start from colder entry regions. Thus, it is expected that gates on the low values of $E_{\gamma_{1}+\gamma_{2}}$, which select cold entry region, should enhance feeding into the high-spin states. Figure (3a) shows a ratio spectrum obtained by dividing the "cold" spectrum of (3b) by the "hot" spectrum of (3c), and illustrates the enhancement factors in a simpie way. The $\gamma$ rays in the $6 \mathrm{n}$ and $5 \mathrm{n}$ channel (squares and triangles, respectively) which are stronger in Fig. (3b) give rise to the largest peaks in the ratio spectrum, indicating largest enhancement factors. In contrast, $\gamma$ rays in the $4 \mathrm{n}$ channel ${ }^{170} \mathrm{Hf}$ which are strong in Fig. (3c) will appear as dips or "inverted peaks" in the ratio spectrum (circles). If the feeding patterns to the highspin states were the same in the two gates, then the enhancement factors would stay constant for the $\gamma$ rays belonging to the same residual nucleus. (Although, they will be different for different exit cahnnels.) In Fig. (3a), it is apparent that the inverted peaks (associated with ${ }^{170} \mathrm{Hf}$ ) deviate significantly from a constant valus First, transitions belonging to the positiveparity yrast sequence (the inverted peaks labeled with filled circles) are nearly a factor of two deeper than those belonging to two of the odd-spin side bands that become the yrast sequence at high spins (labeled with open circles). This implies that feeding of the high-spin yrast states are enhanced by nearly a factor of two when populated from the cold vs. hot entry regions. This is true despite the fact that the higher energy $E_{\gamma_{1}+\gamma_{2}}$ gate populates the hot entry region at slightly higher spins. Interestingly, with increasing spin, the inverted peaks associated with the positive-parity yrast sequence in Fig. (3a) become smaller and indicate that feeding is also enhanced at higher spins within the same band. Thus, by influencing the temperature of the entry region, high-energy $\gamma$ gating exerts a very strong influence on the population of the high-spin states. 


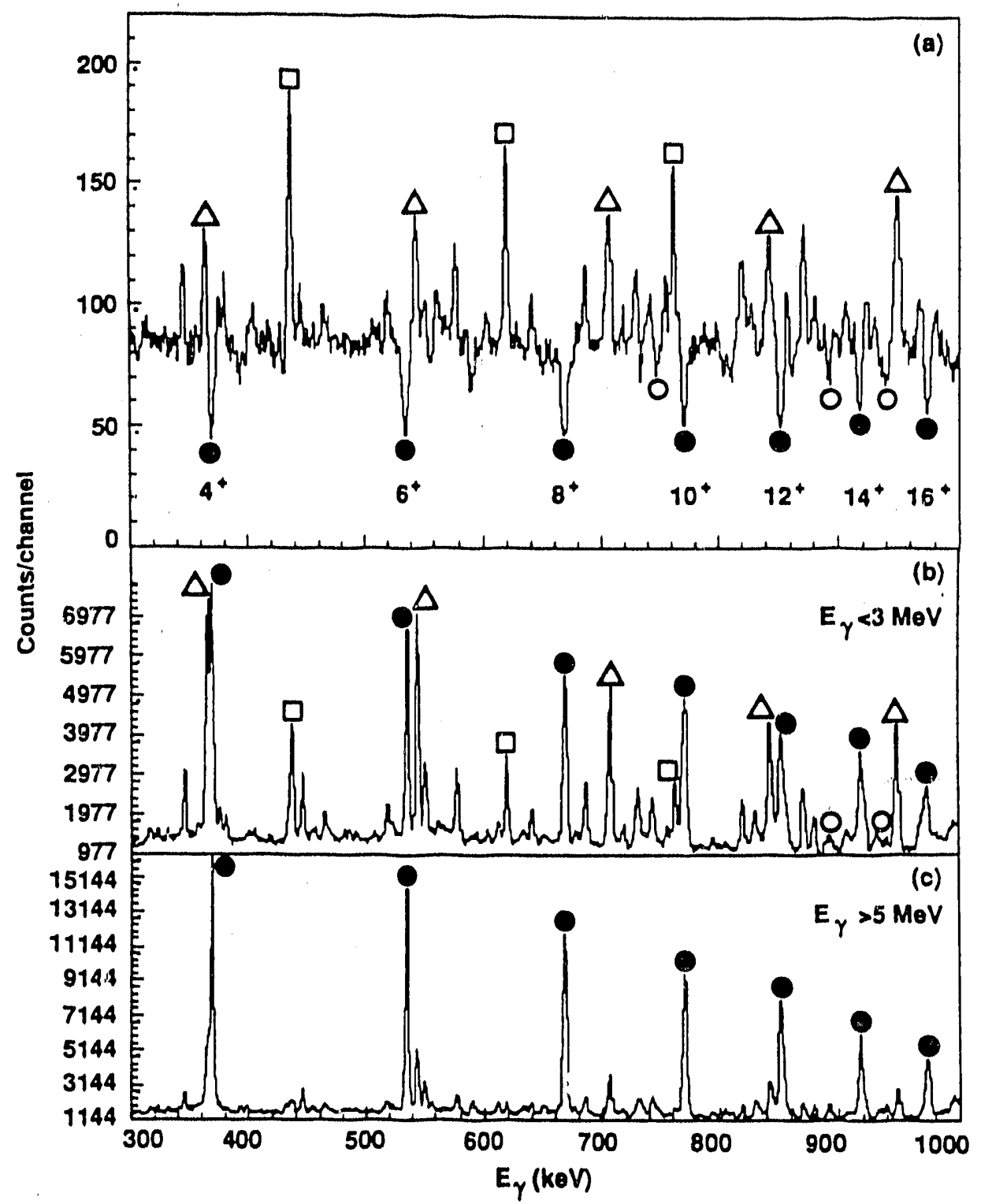

Figure 3: Gamma-ray spectra gated by the high-energy $\gamma$ ray: (c) $E_{\gamma}>5 \mathrm{MeV}$; (b) $E_{\gamma}<3 \mathrm{MeV}$; and (a) the ratio of the two spectra in (b) and (c). The prominent reaction channels identified in the figure are: ${ }^{108} \mathrm{Hf}$ (squares), ${ }^{100} \mathrm{H}_{4}^{\prime}$ (triangles), positiveparity yrast band in ${ }^{170} \mathrm{Hf}$ (filled circles), and negative-parity side bands in ${ }^{170} \mathrm{Hf}$ (open circles).

Recently, there has been extensive discussion of the best conditions under which the disc:ete superdeformed (SD) states are populated. Taras et al. ${ }^{4}$ have reported that the necessary criterion for population of SD bands is to form cold residual nuclei at spins higher than those where SD states become yrast. However, they could not find experimental evidence for the suggestion of Herskind et al..$^{5}$ that, thanks to the enhanced E1 decay probability into the superdeformed minimum, transition through SD states may be enhanced by a factor of 3 by demanding presence of a high-energy $\gamma$ ray $\left(E_{\gamma}>5 \mathrm{MeV}\right)$. Indeed, population of the yrast $\mathrm{SD}$ band in ${ }^{140} \mathrm{Gd}$ was reduced by a. factor 2 when gating on $E_{\gamma}>5 \mathrm{MeV}$ vs. $E_{\gamma}<3 \mathrm{MeV} \gamma$ 
rays, possibly due to the fact that their reaction fed a relatively cold entry distribution.

Our present study offers a natural explanation for the above results. Since the high- and low-energy $\gamma$ gating selects, respectively, hot and cold entry distribution, the SD band will be preferentially populated by the low-energy gate (provided the associated spin distribution remains significantly above the required critical spin). However, this result does not necessarily contradict the validity of the mechanism discussed in Ref. 5 , which can be investigated only after removal of the strong bias that high-energy $\gamma$ gating introduces into the entry-state population. This may be achieved by combining high $E_{\gamma}$ gating with an appropraite $(\mathrm{H}, \mathrm{k})$ gate that would constraint variation of the entry region.

\section{ELECTROMAGNETIC STRENGTH FUNCTIONS}

Deexcitation of the compound nuclei from the entry region down to the ground state takes place via emission of both collective and statistical $\gamma$ rays. Therefore, study of the spintemperature effects in "warm" nuclei requires a detailed knowledge of the electromagnetic strength functions that govern $\gamma$ emission, and directly enter into the Monte Carlo simulations that are used to interpret the experimental data. Almost universally, these statistical models assume constant Weisskopf estimates for the coefficients $C_{O \lambda}$ over the whole deexcitation region. ( $C_{O \lambda}$ determines the strength of gamma emission of mode $O \lambda$.) The simulation calculations then serve to determine the $\left(C_{E_{1}} / C_{E_{2}}\right)$ ratio (which governs the statistical-tocollective competition) from fits to the sidefeeding intensities and lifetimes. However, this simple picture of the gamma strength functions has never been verified experimentally, or theoretically.

Despite the fact that gamma strength functions have been the subject of extensive research for more than three decades, our empirical knowledge remains very limited. The E1 strength function is known only for the low-spins states from neutron-capture gamma rays and giant dipole resonance studies. These studies have revealed that, compared to the Lorentrian strength functon, the E1 strength is suppressed at low $\gamma$ ray energies, and displays a "py $6 \mathrm{~m} . "$ resonance whose resonance energy increases with neutron number for the rare-earth and heavier nuclei. These data have been combined with information from average resonance capture studies to infer an M1 strength of nearly one Weisskopf unit for the $\gamma$ rays that deexcite the low-spin states situated at an excitation energy of nearly one neutron binding energy. ${ }^{7}$

Complementary information about the strength functions at higher spins, and over ithe whole $(U, I)$ map may be obtained from heavy-ion fusion-evaporation reactions. However, a model-independent analysis of data from such reactions has been possible only for the discrete transitions $\left(E_{\gamma}<1 \mathrm{Me} \ddot{v}\right)$ that connect the near-yrast states. In the first detailed study of the continuum $\gamma$ rays using the $(\mathrm{H}, \mathrm{k})$-gating technique at ORNL, it was discovered that, at high spins, the nucleus ${ }^{158} \mathrm{Yb}$ changes from noncollective structures near the yrast line to collective structures near the entry region. ${ }^{8,9}$ This transition is manifested by the onset of a broad collective $\mathrm{E} 2$ (centered at $E_{\gamma} \simeq 1.2 \mathrm{MeV}$ ), and fast energetic M1 (centered at $E_{\gamma} \simeq 2.5$ $\mathrm{MeV}$ ) structures that grow in intensity with increasing excitation energy. The deduced M1 strength of nearly $1 \mathrm{~W} . \mathrm{U}$. is consistent with that measured in neutron-capture studies for the 
low-spin states at comparable excitation energies.

Theoretically, a similarly unexpected result was recently reported by Egido and Weidenmüller who studied spin-temperature dependence of the gamma strength functions using selfconsistent RPA-plus-finite temperature CHFB calculations. ${ }^{10}$ They have concluded that, far from being constant, the electromagnetic strength functions fluctuate widely with spin and excitation energy. Moreover, for $E_{\gamma}<3 \mathrm{MeV}$, the M1 transitions dominate over the the E1 transitions at high excitation energies. Therefore, we have neither experimental, nor theoretical justification to assume that the $E 1$ transitions are primarily responsible for the cooling process, or the gamma strength functions remain constant over the whole (U,I) map. It follows that using oversimplified simulation calculations to interpret the experimental data may oftentimes lead to the wrong conclusions. For example, one can reproduce the feeding pattern of the yrast states using widely different assumptions about the E2 and M1 strength functions, since the cooling process is sensitive to only the ratios of these parameters. ${ }^{11}$

These ambiguities are overcome in the present work which solves a highly overdetermined system. In addition to reproducing the yrast-feeding intensities and lifetimes, we fit the specral shapes of nearly 5000 spectra to determine the collective E2, and statistical E1,M1, and E2 strength functions over 256 grid points that cover the entire $(H, k)$ map. As an additional check, the simulated total dipole and quadrupole spectra that originate from each of these grid points will be compared with the corresponding experimentally determined spectra. This procedure ensures that not only have we determined the correct rat:os of the strength functions for any given pathway, but also their correct absolute values.

Schematically, the analysis involves classification of the grid points according to their intrinsic excitation energies, and fitting the spectral shapes starting with the lowest-lying isotherms. In the spectra of the "first" $\gamma$ rays, the E1 and M1 strengths affect the slope of the high- and low-energy shoulders, respectively. After fitting these slopes, the E2 strength is adjusted to reproduce the correct sidefeeding into the yrast states. The sidefeeding intensities are determined from projections of the Ge spectra subject to the same gating condition. This procedure is then repeated for the isotherms lying successively at higher excitation energies. However, since $\gamma$ decay from these points passes through the colder regions, the spectra for the second, third, ... $\gamma$ rays are once again sensitive to the strength functions associated with the "colder" isotherms. Therefore, the spectra from the higher-lying isotherms provide additional constraints on the strength functions of the lower grid points. Hence, the sysuem being overcostrained by a factor of nearly five, is ensured to be free from having a multitude of solutions. The simulations are being carried out using the GAMBLE rode of Leander ${ }^{12}$, which allows for a variety of E1 strength functions, rotational damping widths, and experimentally determined de ector response functions and coincidence gating conditions.

\section{ROTATIONAL DAMPING}

As discussed by Bent Herskind in the previous talk, it is now estimated that rotational damping sets in at excitation energies of $U \simeq 1-2 \mathrm{MeV}$ above the yrast line. This fragments the $B(E 2)$ transition strength that connects the collective states at spins I and (I-2), and gives 
it a Gaussian distribution with a FWHM value of $\Gamma_{\text {rot }}(U, I)$. The variation of the rotational damping width with spin and temperature has been studied by the Copenhagen group ${ }^{13}$, which has predicted an initial rise in $\Gamma_{\text {rot }}$ with increasing $U$, followed by a fall off of $\Gamma_{\text {rot }}$ at higher excitation energies. ${ }^{14}$ The fall off in $\Gamma_{\text {rot }}$ is analogous to the motional narrowing phenomenon that has been observed in nuclear magnetic' resonance. It would be interesting to investigate the evolution of $\Gamma_{\text {rot }}$ with excitation energy in order to verify the presence of motional narrowing in the more complicated nuclear systems.

As mentioned by Weidenmüller at this conference, there may be some connection between the order-to-chaos transition and the spreading width, $\Gamma_{\mu}$, of the E2 strength function. ${ }^{16,16}$ Recent quntitative calculations by $\AA$ berg show a close parallelism between the chaoticity parameter and the fragmentation of the E2 strength. ${ }^{17}$ Therefore, experimental determination of the fragmentation of the E2 strength as a function of excitation energy may provide a possible probe of the chaoticity of nuclear systems at finite temperatures.

Current studies of $\Gamma_{\text {rot }}$ revolve around determination of the loss of energy correlation in high-resolution double- and triple-gamma coincidence data. ${ }^{18,10}$ In this approach, $\Gamma_{\text {rot }}$ values are determined as a function of $\gamma$ ray energies, and reflect the average damping width along a pathway that becomes increasingly more hot as the $\gamma$ ray energy increases. They, therefore, lack thermal specificity.

In the present analysis, following the emission of the first few $\gamma$ rays which are dominated by the high-energy statistical transitions, the energy-ordered $\gamma$ rays assume a nearly Gaussian shape (e.g., see the $\mathrm{N}=10$ spectrun in Fig. 1). The FWHM of this distribution is partly due to the detector response function, and partly due to the damping width. Therefore, after allowance has been made for the instrumental effects, and the statistical transitions that give rise to the shoulders, the remaining width is attributed to the $\Gamma_{\text {rot }}$. The temperature effects on $\Gamma_{\text {rot }}$ may then be studies by moving the $(H, k)$ grid point to higher excitation energies.

Figure 4 shows the FWHM of the raw, uncorrected data for three excitation energies $(\mathrm{U}=2,4$, and $8 \mathrm{MeV})$. For comparison, $\Gamma_{\text {rot }}$ values obtained from $\gamma-\gamma$ energy correlation analysis of ${ }^{170} \mathrm{Hf}$ (diamonds) at $\mathrm{ORNL} \mathrm{L}^{2}$, and ${ }^{108} \mathrm{Hf}$ (open squares) at $\mathrm{NBI}^{20}$ are also shown. The filled square reprents $\Gamma_{\text {rot }}$ value for ${ }^{160} \mathrm{Yb}$ obtained from a triple- $\gamma$ correlation analysis by the NBI group. ${ }^{20}$ Eecause of the detector response function (e.g., fiuite resolution, misordering due to finite solid angle and detection efficiency, and a continuum of Compton background), as well as the broadening due to the statistical transitions which increases with increasing temperature, the plotted FWHM values represent an upper bound on $\Gamma_{\text {rot. }}$. Although it is difficult to draw firm conclusions about the evolution of $\Gamma_{\text {rot }}$ with excitation energy from these uncorrected data, it is possible to get some qualitative estimates. Since the lowest curve corresponds to a very cold system $(\mathrm{U}=2 \mathrm{MeV})$, it may bia taisen as the reference to represent all the instrumental effects. We can then crudely estimate $\Gamma_{\text {rot }}$ at $U=4$ and $8 \mathrm{MeV}$. These values are of the same order of magnitude as those obtained from $\gamma \gamma$ correlation analysis, and grow slower than $\sqrt{T}$ which is the expected dependence in the absence of motional narrowing. Naturally, a full analysis of the data is needed to confirm these preliminary trends. 


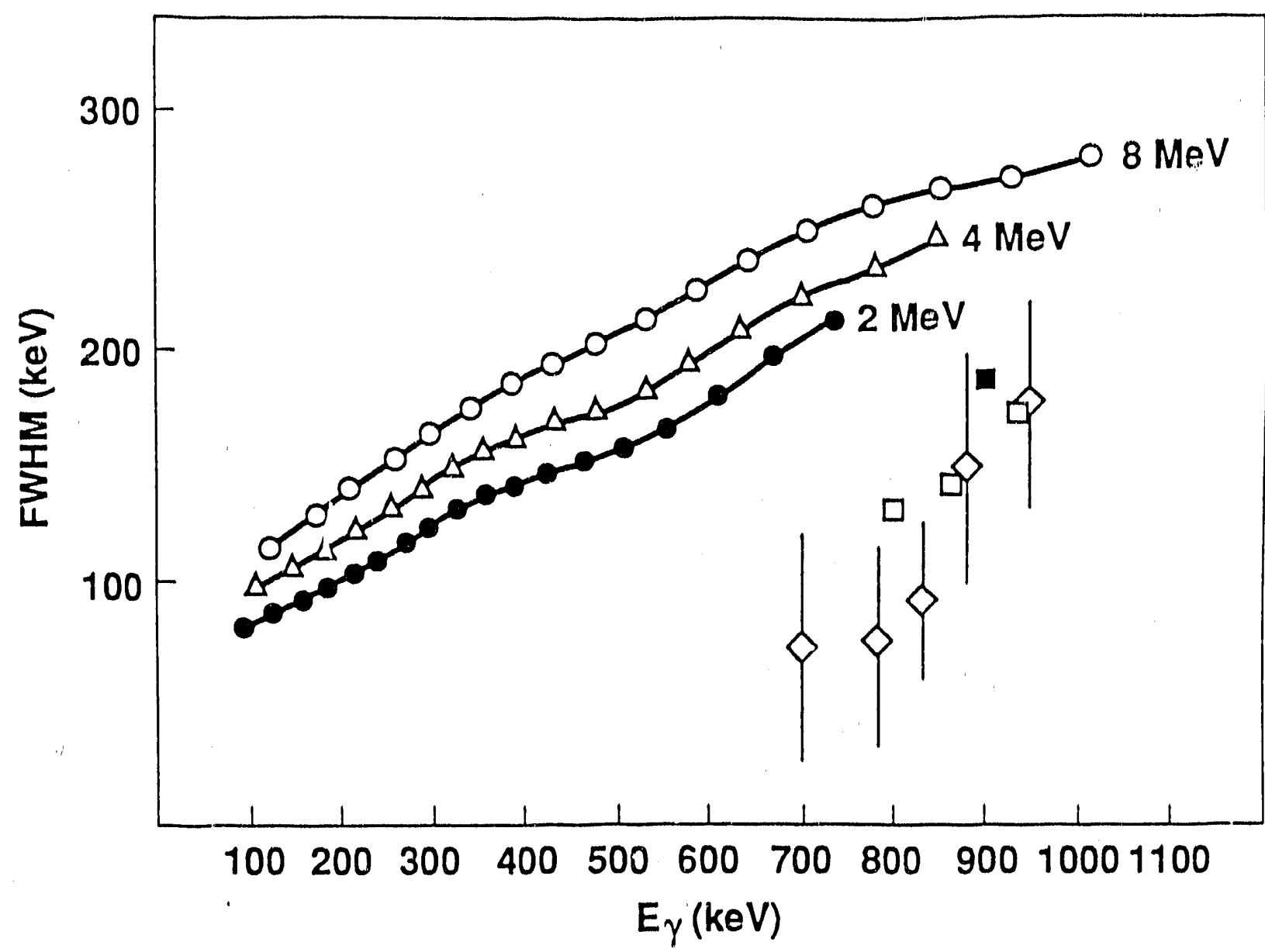

Figure 4: The evolution of the FWHM of the histograms of the energy-ordered $\gamma$ rays as a function of their centroids for entry states at 2,4 , and $8 \mathrm{MeV}$ excitation energy. They represent an upper bound on the $\Gamma_{\text {rot }}$ values. For comparison, $\Gamma_{\text {rot }}$ values obtained from $\gamma-\gamma$ energy correlation studies of the neighbouring nuclei are also shown (see text).

\section{SUMIIARY}

Nuclei are expected to undergo dramatic structural changes at finite temperatures. Onset of chaos, damping of the rotational motion, pairing phase transition, shape cisanges, and melting of the shell structure are a few of the fascinating topics to be explored in warm nuclei. Unfortunately, Technical difficulties have seriously impeded our experimental studies of these finite-temperature effects. With the prospects of the next generation of powerful $\gamma$-detection systems in mind, we have investigated a new continuum $\gamma$-ray spectroscopy technique that opens up the possibility of addressing some of these problems. This technique allows determination of gamma strength functions, rotational damping widths, and $J^{(2)}$ moments of inertia as a fuction of spin and temperature. The preliminary results indicate that $\Gamma_{\text {rot }}(U)$ increases slower than $U^{(1 / 4)}$, which suggests presence of some saturation effect. The technique of highenergy $\gamma$ gating may also be applied to isolate either "hot", or "cold" regions of the entry state for finite-temperature effect studies. As a result, it provides a powerful filter to select exit-channels of interest, or to significantly enhance feeding of the near-yrast discrete states by populating the cold entry states. This has important implications for the study of feeding 
into the superdeformed states.

Oak Ridge National laboratory is operated by Martin Marietta Energy Systems, Inc. for the U.S. Department of Inergy under contract No. DE-AC05-84OR2140n.

\section{References}

1) M. Jääskeläinen $e \nmid$ al, Nucl. Instr. Meth. 204 (1983) 385.

2) I. Y. Lee et al., in Proc. Conference on High-Spin Nuclear Structure and Novel Shapen, Argonne National Laboratory, April 1988, Report ANL-PHY-88-2, pp. 160; and to be published.

3) M. Guttormsen et al, Nucl. Inst. Meth. A255 (1987) 518.

4) P. Taras et al. , Phys. Rev, Lett. 61 (1988) 1348.

5) B. Herskind et al', , Phys. Rev. Lett. 59 (1987) 2416; K. Schiffer and B. Herskind, this volume.

6) G. A. Bartholornew et al, in: Advances in Nuclear Physics, eds. M. Baranger and E. Vogt (Plenum Press, New york, 1973) pp. 229.

7) D. Gardner et al, in: Fifth International Symposium on Cupture Gamma-Ray Spectroscopy and Related Topics, 1984 AIP Conference Proceedings No. 125, pp. 513.

8) C. Baktash et al., Phys. Rev. Lett. 54978 (1985).

9) C. Baktash, in: The Variety of Nuclear Shapes, eds. J. D. Garrett et al. (World Scientific, Singapore, 1988) pp. 537.

10) J. L. Egido and H. A. Weidenmüller, Phys. Lett, 208B, (1988) 58; J. L. Egido and H. A. Weidenmüller, Phys, Rev. C 30 (1989) 2398.

11) G. Leander, Phys. Rev. C 38 (1938) 728.

12) G. Leander, Comput. Phys. commun. 47 (1987) 311.

13) B. Lauritzen et al., Nucl. Phys. A457 (1986) 61.

14) R. A. Boglia et al. , Phys. Rev. Lett. 58 (1987) 326.

15) T. Guhr and H. A. Weidenmüller, Ann. Phy。. (N.Y.) 193 (1989) 472.

16) T. H. Seligman et al., Phys. Lett. 168b (1986) 365.

17) S. Åberg, preprint 1990.

18) J. Bacelar et al., Phys. Rev. Lett. 55 (1985) 1858.

19) F. S. Stephens et al., Phys. Rev.Lett. 58 (1987) 2186.

20) B. Herskind, in: Slide Report of the Workshop on Nuclear Structure in the Era of New Spectroscopy, Part B, The Niels Bohr Institute, October 1989, pp.257 

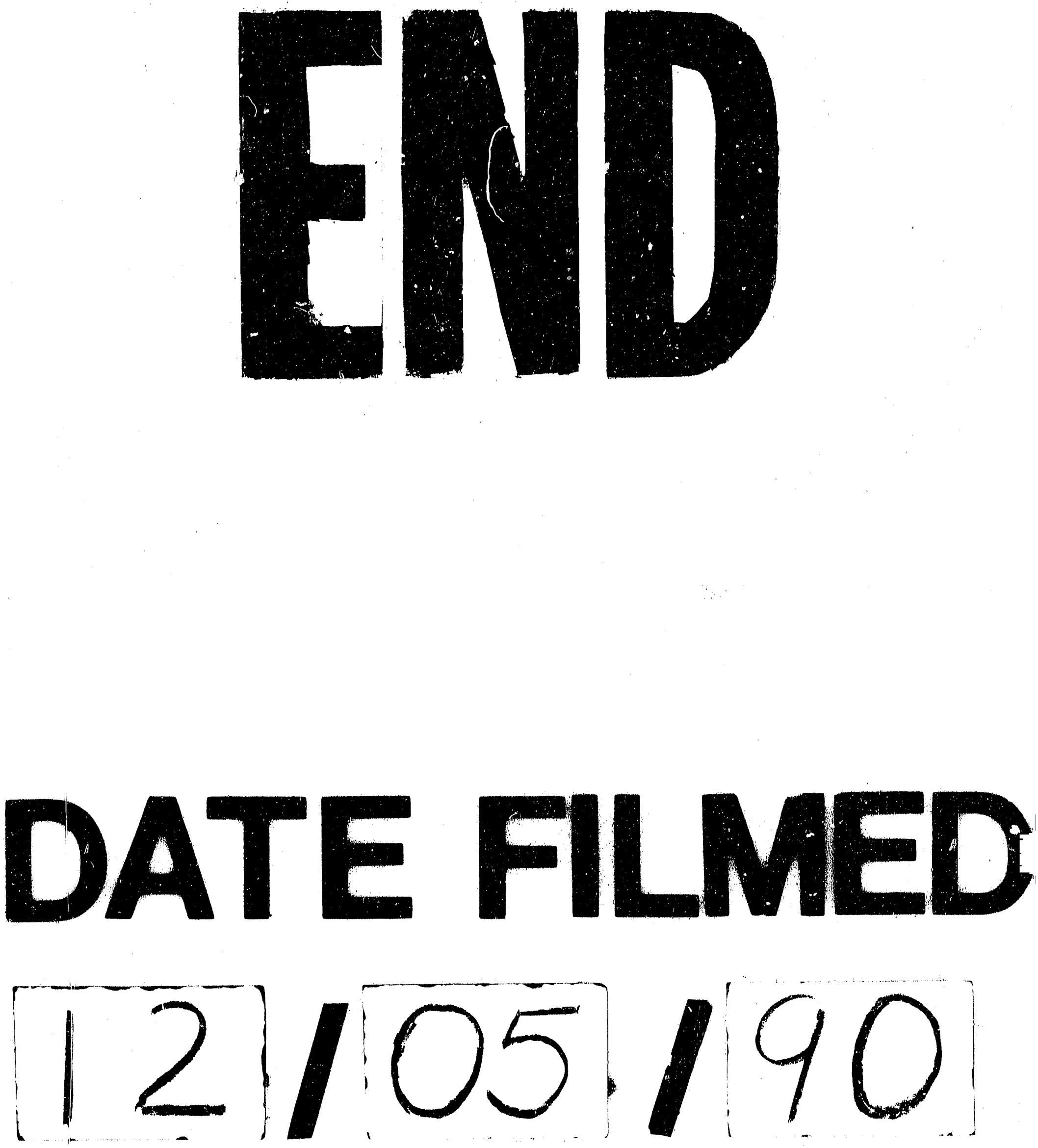
